\title{
THOMPSONELLA NELLYDIEGOAE (CRASSULACEAE), UNA NUEVA ESPECIE DEL SUR DE MÉXICO
}

\author{
Pablo Carrillo-Reyes ${ }^{1}$ y Emmanuel Pérez-Calix ${ }^{2}$
}

\begin{abstract}
${ }^{1}$ Instituto de Ecología, A.C., Departamento de Biología Evolutiva, km. 2.5 Carretera antigua a Coatepec, 91070 Xalapa, Veracruz, México. pcarreyes@gmail.com ${ }^{2}$ Instituto de Ecología, A.C., Centro Regional del Bajío, Apdo. postal 386, 61600 Pátzcuaro, Michoacán, México. emmanuel.perezcalix@inecol.edu.mx
\end{abstract}

\section{RESUMEN}

Con base en colecciones realizadas en los estados de Guerrero, Oaxaca y Puebla, se describe como especie nueva y se ilustra Thompsonella nellydiegoae. Se le compara con Thompsonella platyphylla Rose, la cual parece ser la especie más cercanamente relacionada, de la que se distingue por sus inflorescencias robustas de hasta $1 \mathrm{~m}$ de altura con cincinos compactos de hasta 10 flores y por tener márgenes de las hojas rojizos y crenulados. También se compara con T. colliculosa Moran y con T. garcia-mendozae P. Carrillo et Pérez-Calix, con las que presenta algunas semejanzas morfológicas.

Palabras clave: Crassulaceae, Guerrero, México, Oaxaca, Puebla, Thompsonella.

\begin{abstract}
Based on collections made in the Mexican states of Guerrero, Oaxaca and Puebla, Thompsonella nellydiegoae is described as new species and illustrated. It is compared with Thompsonella platyphylla Rose, which seems to be the closest related species and can be distinguished by its large inflorescences up to $1 \mathrm{~m}$ high, with compact cincinni of up to 10 flowers and by having leaves with red and crenulate margins. It is also compared with $T$. colliculosa Moran and T. garcia-mendozae P. Carrillo et Pérez-Calix, with which it shares some morphological features.
\end{abstract}

Key words: Crassulaceae, Guerrero, Mexico, Oaxaca, Puebla, Thompsonella. 
Durante la revisión de herbario y los viajes de colecta encaminados a realizar una revisión taxonómica de Thompsonella, se detectó la existencia de algunas plantas de dudosa identidad en este género, una de las cuales ya ha sido dada a conocer como especie nueva (Carrillo-Reyes y Pérez-Calix, 2006). El conocimiento sobre la existencia de otro de estos elementos fue posible a partir de varias recolectas producto de la intensa exploración que recientemente ha sido efectuada en el estado de Guerrero por el equipo de trabajo coordinado por la Maestra Nelly Diego Pérez de la Facultad de Ciencias de la Universidad Nacional Autónoma de México. Tras realizar varios viajes a la parte noreste de Guerrero se llevaron a cabo colectas adicionales y se hicieron observaciones de campo que permitieron concluir que se trata de una especie no descrita, misma que aquí damos a conocer como:

Thompsonella nellydiegoae P. Carrillo et Pérez-Calix sp. nov. Fig. 1.

Planta perennis, glabra, pruinosa; folia in rosula, oblanceolata, 7-15(-17) $\mathrm{cm}$ longa, 3-5.8 cm lata, apice obtusa, margine crenulata rubra; inflorescentia thyrsiformis, (30-)40-60(-100) cm longa, cincinni 1-2 cm longi, flores in cincinno 4-10 (-12), ca. 8-9 mm diametro; sepala inaequalia, lanceolato-ovata, 2.5-4 $\mathrm{mm}$ longa, 1.2-2 mm lata, apice acuta ad obtusa.

Planta herbácea perenne, glabra, hasta de $100 \mathrm{~cm}$ de altura (incluyendo la inflorescencia); tallo hasta de $5.5 \mathrm{~cm}$ de largo y $2.4 \mathrm{~cm}$ de diámetro, subterráneo o aéreo, suculento, densamente cubierto por raíces adventicias de color cobrizo; hojas formando una roseta hasta de 10 a $15(-20) \mathrm{cm}$ de diámetro, ascendentes, oblanceoladas, de 7 a 15(-17) cm de largo, de 3 a $5.8 \mathrm{~cm}$ de ancho y de $1 \mathrm{~cm}$ de grueso cerca de la base, ápice obtuso, base semi-abrazadora, glabras, glaucas, de color verde claro grisáceo con márgenes rojizos, crenados; inflorescencias de (30-)40 a $60(-100) \mathrm{cm}$ de largo, tirsoides con (25-)30 a 80 o más ramas laterales en forma de cincinos de 1 a $2 \mathrm{~cm}$ de largo y con 4 a 10(-12) flores cada uno; pedúnculo erecto, de 20 a $42 \mathrm{~cm}$ de largo y hasta de $1.4 \mathrm{~cm}$ de grueso cerca de la base; brácteas hasta de $1.5 \mathrm{~cm}$ de largo y $0.5 \mathrm{~cm}$ de ancho; flores de 8 a $9 \mathrm{~mm}$ de diámetro; pedicelos hasta de $1 \mathrm{~mm}$ de largo; sépalos desiguales, anchamente lanceolados a ovados, de 2.5 a $4 \mathrm{~mm}$ de largo, de 1.2 a $2 \mathrm{~mm}$ de ancho, hasta de $1.4 \mathrm{~mm}$ de grueso, farinosos, suculentos, de color verde olivo, la superficie interna con manchas rojizas irregulares, ápice agudo a obtuso con un pequeño mucrón rojizo; pétalos lanceoladoelípticos, de 5 a $6 \mathrm{~mm}$ de largo, de ca. $2 \mathrm{~mm}$ de ancho, ápice acuminado, base atenuada, cara adaxial blanca, con líneas pardo-rojizas en los tres cuartos distales, 


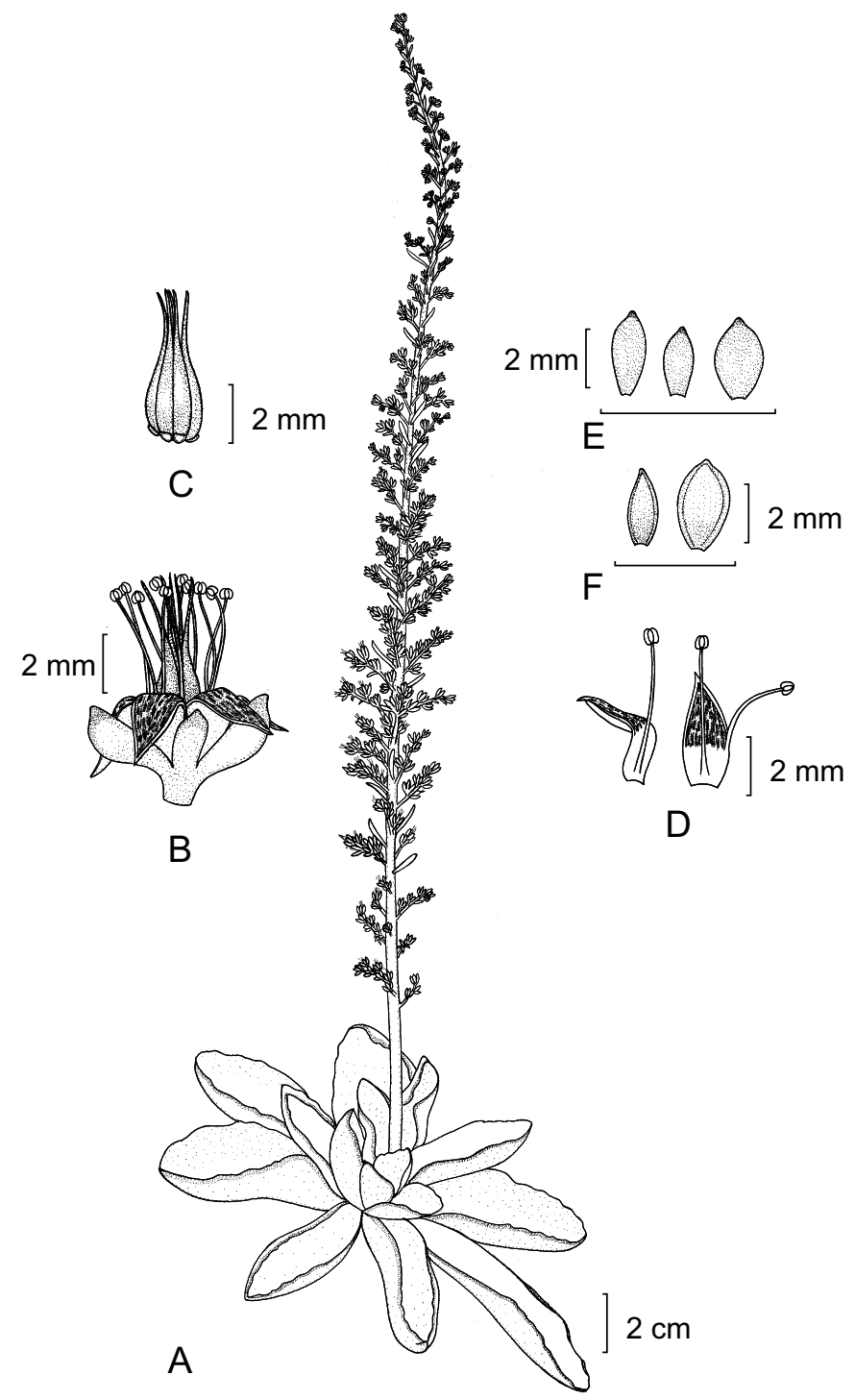

Fig. 1. Thompsonella nellydiegoae. A. hábito; B. flor; C. gineceo; D. pétalos; E. sépalos en vista abaxial; F. sépalos en vista adaxial. 
envés pruinoso; estambres de 5 a $6 \mathrm{~mm}$ de largo, filamentos de 4.5 a $5.5 \mathrm{~mm}$, de color verde-amarillento brillante, anteras de ca. $0.4 \mathrm{~mm}$ de largo, de ca. $0.5 \mathrm{~mm}$ de ancho, rojizas, amarillas en dehisencia; nectarios de ca. $0.4 \mathrm{~mm}$ de largo; gineceo de 6.5 a $7 \mathrm{~mm}$ de largo, de ca. $2.4 \mathrm{~mm}$ de ancho, de color verde-amarillento brillante, glabro, estigma inconspicuo; folículos piriformes, erectos, de hasta $7 \mathrm{~mm}$ de largo, incluyendo el estilo de ca. $1.5 \mathrm{~mm}$ de largo y hasta de $1.2 \mathrm{~mm}$ de ancho, de color café rojizo; semillas de 0.6 a $0.7 \mathrm{~mm}$ de largo, de ca. $0.2 \mathrm{~mm}$ de ancho, de color café.

Tipo: México, Guerrero, municipio de Olinalá, $1.3 \mathrm{~km}$ al E de Olinalá por el camino a Cualac. Alt. $1320 \mathrm{~m}$. Rocas calizas, bosque tropical caducifolio. P. Carrillo-Reyes, A. Abundis y F. Z. Vaz-de-Mello 4907 (Holotipo: IEB, isotipos: MEXU, $\mathrm{XAL})$.

Paratipos: México, Guerrero: municipio de Chilapa de Álvarez, Chilapa, sobre tejados, P. Carrillo-Reyes y M. I. Chacón 3679 (GUADA); Cañada del Peral, $P$. Carrillo-Reyes y M. I. Chacón 3677 (GUADA); municipio de Copalillo, $5 \mathrm{~km}$ al S de Papalutla rumbo a Chimalacacingo, M. Martínez G. 597 (FCME); municipio de Olinalá, $1 \mathrm{~km}$ al $\mathrm{N}$ de Tecolapa por el camino Olinalá - Tecolapa, J. L. Contreras 2441 (FCME); Coachimalco, sobre tejados, P. Carrillo-Reyes y M. I. Chacón 3681 (GUADA); Olinalá, sobre tejados, P. Carrillo-Reyes, E. Pérez y L. Ortiz-Catedral 2743 (IEB); $1.3 \mathrm{~km}$ al E de Olinalá, P. Carrillo-Reyes y M. I. Chacón 3683 (GUADA, IEB); municipio de Cualac, Cualac, sobre tejados, P. Carrillo-Reyes, E. Pérez y L. Ortiz-Catedral 2744 (IBUG, IEB); municipio de Tlapa de Comonfort, Tlapa, sobre tejados, P. Carrillo-Reyes y M. I. Chacón 3680 (GUADA), Oaxaca: Distrito de Juxtlahuaca, municipio de San Miguel Tlacotepec, Sabanillas, M. Cházaro, M. Kimnach $y$ M. Negrete 7111 (CAS, IBUG, IEB, MEXU, WIS, XAL); $5 \mathrm{~km} \mathrm{~N}$ of San Miguel Tlacotepec, M. Kimnach, M. Cházaro y M. Negrete 3207 (MEXU); municipio de San Juan Mixtepec, Caba Coo, 1 km al N de San Juan Mixtepec, J. Reyes 1035 (IEB).

Etimología: Se dedica el nombre de esta especie a la Maestra Nelly Diego Pérez, de la facultad de Ciencias, de la Universidad Nacional Autónoma de México, quien ha contribuido de manera notable al conocimiento de la flora del estado de Guerrero.

Distribución y hábitat: Se le conoce de varias localidades del norte de Guerrero, noroeste de Oaxaca y suroeste de Puebla, donde crece en afloramientos de roca caliza en el bosque tropical caducifolio, bosques de Juniperus y en encinares. Es común 
también encontrarla formando colonias sobre los tejados de las casas dentro de su área de distribución (Fig. 2). Se le encuentra en altitudes que van de 900 a 1900 m.

Durante la preparación de este trabajo, uno de los autores fue alertado a través del envío de fotografías, sobre la existencia de esta especie en el suroeste de Puebla (municipio de Teotlalco), desgraciadamente no se observaron especimenes de herbario de esa localidad.

Fenología: Florece de julio a noviembre. Fructifica a partir de septiembre.

Thompsonella nellydiegoae es una planta robusta que se caracteriza por tener las ramas secundarias de la inflorescencia en forma de cincinos con hasta 10 flores, su inflorescencia que generalmente sobrepasa los $50 \mathrm{~cm}$ de largo, así como por sus hojas gruesas, glaucas, acanaladas en la base y con márgenes crenulados y rojizos (Fig. 2). Carrillo-Reyes et al. (en prensa) han encontrado evidencia de que Thompsonella platyphylla Rose es la especie más cercanamente relacionada al nuevo taxon. Ambas prosperan en el bosque tropical caducifolio de la cuenca alta del Río Balsas (Río Mixteco), sobre sustrato calizo, sin embargo no se tiene noticia de ninguna localidad en la que sean simpátricas. Aunque las dos tienen hojas glaucas, en T. nellydiegoae los márgenes de las mismas son crenulados y rojizos, mientras que en T. platyphylla son enteros y acaso ligeramente más oscuros que el resto de la lámina. Otros caracteres que comparten son los cincinos con numerosas flores y los tallos cubiertos por raíces cobrizas; sin embargo, en T. nellydiegoae los cincinos son más compactos y las inflorescencias son notablemente más robustas; ninguna otra especie de Thompsonella tiene inflorescencias que lleguen a superar los $60 \mathrm{~cm}$ de longitud (Moran, 1992). Otras representantes del género que portan cincinos con numerosas flores son T. colliculosa Moran y T. garcia-mendozae P. Carrillo et Pérez-Calix, sin embargo a diferencia de la nueva entidad, en esas especies las inflorescencias son más laxas, y las líneas en la cara adaxial de los pétalos en ocasiones llegan a ser tan densas que hacen ver su superficie expuesta totalmente roja.

\section{AGRADECIMIENTOS}

Agradecemos a Jerzy Rzedowski y a dos revisores anónimos los comentarios al manuscrito. Alejandro Abundis-Santamaría, María Isabel Chacón, Luis Ortiz-Catedral y Fernando Z. Vaz-de-Mello nos ayudaron y acompañaron durante el trabajo 

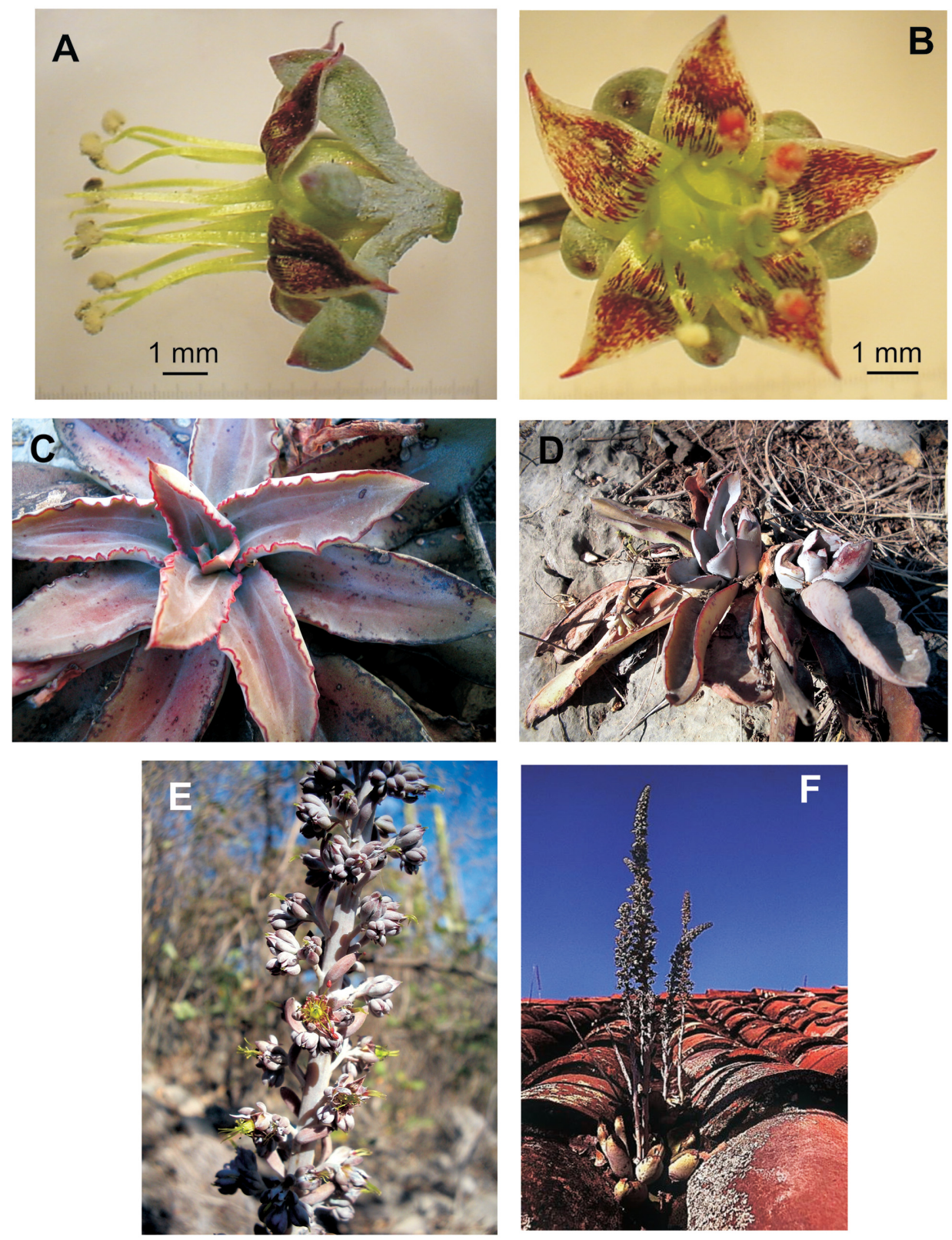

Fig. 2. Thompsonella nellydiegoae. A. flor en vista lateral; B. flor vista de arriba; C. detalle de la roseta; D. hábito; E. detalle de la inflorescencia; F. hábito. 
de campo. Agradecemos también a Rogelio Cárdenas Soriano la elaboración del dibujo; a Gabriel Téllez-Torres por informarnos sobre su colecta del estado de Puebla; y a los curadores de los herbarios CAS, FCME, GUADA, IBUG, IEB, MEXU, WIS y XAL por permitirnos consultar sus colecciones.

\section{LITERATURA CITADA}

Carrillo-Reyes, P. y E. Pérez-Calix. 2006. Una nueva especie de Thompsonella (Crassulaceae) del estado de Michoacán, México. Novon 16: 320-323.

Carrillo-Reyes, P., V. Sosa y M. Mort. (en prensa). Thompsonella and the "Echeveria group" (Crassulaceae): phylogenetic relationships based on molecular and morphological characters. Taxon.

Moran, R. 1992. Thompsonella Britton \& Rose (Crassulaceae), with T. colliculosa, a new species. Cact. Succ. J. (L.A.) 64: 37-44.

Recibido en enero 2008.

Aceptado en abril 2008. 
